\title{
Revisiting the Marton, Simpson, and Suddeth experimental confirmation of the Aharonov-Bohm effect
}

\author{
James Macdougall* and Douglas Singleton + \\ Physics Department, CSU Fresno, Fresno, CA 93740 USA \\ Elias C. Vagenast \\ Theoretical Physics Group, Department of Physics, \\ Kuwait University, P.O. Box 5969, Safat 13060, Kuwait
}

(Dated: July 17, 2018)

\begin{abstract}
We perform an "archeological" study of one of the original experiments used as evidence for the static, time-independent Aharonov-Bohm effect. Since the experiment in question [1] involved a time varying magnetic field we show that there are problems with the explanation of this experiment as a confirmation of the static Aharonov-Bohm effect - specifically the previous analysis ignored the electric field which arises in conjunction with a time-varying magnetic flux. We further argue that the results of this experiment do in fact conform exactly to the recent prediction [2, 3] of a cancellation between the magnetic and electric phase shifts for the time-dependent Aharonov-Bohm effect. To resolve this issue a new time-dependent Aharonov-Bohm experiment is called for.
\end{abstract}

\footnotetext{
*Electronic address: jbm34@mail.fresnostate.edu

$\dagger$ Electronic address: dougs@csufresno.edu

${ }^{\ddagger}$ Electronic address: elias.vagenas@ku.edu.kw
} 
The experimental work of Chambers [4] is usually cited as the first experimental confirmation of the Aharonov-Bohm (AB) effect [5, 6]. However, the earliest (inadvertent) test of the AB effect was an experiment by Marton, Simpson, and Suddeth (MSS) [1], whose set-up was designed simply to make an electron interferometer to see electron interference fringes, and this was apparently accomplished; interference fringes were seen. However, it was realized later that the electrons in [1] were moving through a stray $60 \mathrm{~Hz}$ magnetic field. Since MSS did see a fixed interference pattern, it was originally suggested that the effect of this magnetic field could be ignored given the $60 \mathrm{keV}$ kinetic energy of the electrons. But with the proposal of the AB effect [5], it was realized that there should have been a time-varying shifting of the interference pattern which was not seen in [1]. Thus, the non-observation by MSS of a time-varying, shifting interference pattern appeared to be evidence against the $\mathrm{AB}$ effect. However, in a clever paper Werner and Brill [7] proposed that if one took into account the additional phase shift due to the direct Lorentz force, that under certain conditions there would be an almost exact cancellation of the AB phase shift versus the phase shift coming from, $q\left(\frac{\mathbf{v}}{c} \times \mathbf{B}\right)$. Thus, according to Werner and Brill, the fact that MSS saw a fixed interference pattern could be taken as evidence of the phase shift predicted by the AB effect.

However, the analysis of [7] ignored the electric field which arises whenever there is a time-varying magnetic field. From Faraday's law, i.e., $\nabla \times \mathbf{E}=-\frac{1}{c} \partial_{t} \mathbf{B}$, one can see that there was an electric field present in the MSS experiment. In terms of potentials, the time-varying magnetic field is associated with a time-varying vector potential, namely $\mathbf{B}(\mathbf{x}, t)=\nabla \times \mathbf{A}(\mathbf{x}, t)$. It is this time-varying vector potential that produces an electric field $\mathbf{E}=-\frac{1}{c} \partial_{t} \mathbf{A}(\mathbf{x}, t)$. Now, in [2, 3] a simple argument was given that the phase shift of this electric field would always exactly cancel the AB phase shift coming from the magnetic flux. Briefly, the argument of [2, 3] went as follows: in terms of the electric and magnetic fields one can write the $\mathrm{AB}$ phase shift, $\alpha$, as

$$
\alpha=\frac{e}{\hbar} \int_{C} F=\frac{e}{\hbar}\left[\int \mathbf{E} \cdot d \mathbf{x}(c d t)+\int \mathbf{B} \cdot d \mathbf{S}\right],
$$

where $F$ is the Faraday two-form which in wedge notation is $\left(E_{x} d x+E_{y} d y+E_{z} d z\right) \wedge(c d t)+$ $B_{x} d y \wedge d z+B_{y} d z \wedge d x+B_{z} d x \wedge d y$. In the far right-hand side of Eq. (11) we have reverted to 3-vector notation. Now, for the time-dependent Aharonov-Bohm set-up, i.e., a solenoid with a time-varying flux, one has a time-varying vector potential, $\mathbf{A}(\mathbf{x}, t)$, from which one 
can obtain the fields via $\mathbf{E}=-\frac{1}{c} \partial_{t} \mathbf{A}$ and $\mathbf{B}=\nabla \times \mathbf{A}$. As pointed out in [2, 3], doing the time integration for the electric part of Eq. (11) yields

$$
\int \mathbf{E} \cdot d \mathbf{x}(c d t)=-\oint \mathbf{A} \cdot d \mathbf{x}
$$

and using Stokes' theorem on the magnetic part of Eq. (11) yields

$$
\int \mathbf{B} \cdot d \mathbf{S}=\oint \mathbf{A} \cdot d \mathbf{x}
$$

Substituting the electric and magnetic parts, Eq. (2) and Eq. (3), respectively, in Eq. (10), the total phase shift will be zero, i.e., $\alpha=0$. This was the prediction for the time-dependent AB effect from [2, 3] and this is in fact what was seen in the MSS experiment. In [2] this cancellation between the was also shown using the path length difference (and therefore phase difference) produced in the electron due to the acceleration coming from $\mathbf{E}$.

There are several comments to make about the above results: First the above argument only implies a canceling of the time-dependent part of the phase shift not the timeindependent part,i.e., the part normally referred to as the AB phase shift. If one splits the vector potential in static and time-varying parts as $\mathbf{A}(\mathbf{x}, t)=\mathbf{A}_{0}(\mathbf{x})+\mathbf{A}_{1}(\mathbf{x}, t)$, it is only the contribution coming from $\mathbf{A}_{1}(\mathbf{x}, t)$ which will cancel between Eq. (2) and Eq. (3). In detail $\mathbf{B}=\nabla \times \mathbf{A}_{0}(\mathbf{x})+\nabla \times \mathbf{A}_{1}(\mathbf{x}, t)$ while $\mathbf{E}=-\partial_{t} \mathbf{A}_{1}(\mathbf{x}, t)$. Thus it is only the time-dependent part of the vector potential $\mathbf{A}_{1}(\mathbf{x}, t)$ which takes part in the cancellation between (2) and (3) - the time-independent part $\mathbf{A}_{0}(\mathbf{x})$ still leads to the usual static AB phase shift. This interplay between the electric and magnetic contributions found in Eq. (2) and Eq. (3) was noted in a different context in Ref. [8] (see also [9]). In this paper the cancellation between Eq. (2) and Eq. (3) is shown without using the potentials but rather is shown to be a direct consequence of Faraday's Law which in form notation reads $d F=0$. In detail van Kampen noted that the loop integral of the electric field could be turned into a surface integral via Stokes' theorem as

$$
\oint \mathbf{E} \cdot d \mathbf{x}=\int \nabla \times \mathbf{E} \cdot d \mathbf{S}=-\frac{1}{c} \int \partial_{t} \mathbf{B} \cdot d \mathbf{S},
$$

where in the last step we have used Faraday's law $\nabla \times \mathbf{E}=-\frac{1}{c} \partial_{t} \mathbf{B}$. Now applying $\int \ldots(c d t)$ to (4) we obtain

$$
\int(\oint \mathbf{E} \cdot d \mathbf{x}) c d t=-\int\left(\int \partial_{t} \mathbf{B} \cdot d \mathbf{S}\right) d t=-\int \mathbf{B} \cdot d \mathbf{S}
$$


where the time integral has been undone via the time integration, and we see that this last expression in (5) cancels the expression in (3). Thus following van Kampen we see that the cancellations is a consequence of the Bianchi identities $d F=0$. Second one can ask about the nature of the space-time surfaces in the expression in (1) versus the surfaces used in the more usually encountered magnetic expression such as in (3). To this end we look at infinitesimal paths and surfaces for the above case and show that the cancellation of the time-dependent electric and magnetic pieces occurs. For a solenoid oriented along the $z$-axis the vector potential inside and outside a solenoid, which has a radius $\rho=R$ and a time varying flux, is given by

$$
\mathbf{A}_{\text {in }}=\frac{\rho B(t)}{2} \hat{\varphi} \quad \text { for } \rho<\mathrm{R} \quad ; \quad \mathbf{A}_{\text {out }}=\frac{B(t) R^{2}}{2 \rho} \hat{\varphi} \quad \text { for } \rho \geq \mathrm{R} .
$$

The associated magnetic $(\mathbf{B}=\nabla \times \mathbf{A})$ and electric fields $\left(\mathbf{E}=\partial_{t} \mathbf{A}\right)$ are

$$
\mathbf{B}_{\text {in }}=B(t) \hat{\mathbf{z}} \quad \text { for } \rho<\mathrm{R} \quad ; \quad \mathbf{B}_{\text {out }}=0 \quad \text { for } \rho \geq \mathrm{R},
$$

and

$$
\mathbf{E}_{\text {in }}=-\frac{\rho \dot{B}(t)}{2} \hat{\varphi} \quad \text { for } \rho<\mathrm{R} \quad ; \quad \mathbf{E}_{\text {out }}=-\frac{\dot{B}(t) R^{2}}{2 \rho} \hat{\varphi} \quad \text { for } \rho \geq \mathrm{R} .
$$

Now using these expressions in (7) (8) for some short time interval $\Delta t$, expanding the magnetic field as $B(t)=B_{0}+\dot{B} \Delta t+\mathcal{O}(\Delta t)^{2}$, and taking the path to be a short circular arc segment covered in time $\Delta t$ i.e. $\Delta \mathbf{x}=(\rho \Delta \varphi) \hat{\varphi}$ we find that the small phase for the infinitesimal path and associated area are

$$
\Delta(\alpha)=\frac{e}{\hbar}(\mathbf{E} \cdot \Delta \mathbf{x} \Delta t+\Delta \mathbf{B} \cdot \Delta \mathbf{S})=\frac{e}{\hbar}\left(-\frac{R^{2}}{2 \rho}(\dot{B} \Delta t)(\rho \Delta \varphi)+\frac{\Delta \varphi R^{2}}{2}(\dot{B} \Delta t)\right)=0 .
$$

Thus one can see the cancellation between the electric and magnetic pieces in (9) for this infinitesimal path and area. Again this cancellation just involves the time-dependent part i.e. the $\dot{B} \Delta t$ part of the magnetic field expansion. The constant first term, $B_{0}$, would still lead the time-independent, static AB phase shift.

We now move on to the re-analysis of the MSS experiment. Originally the non-observation of a time shifting interference pattern by MSS led to the idea that this experiment had ruled out the AB effect. However, in [7] Werner and Brill argued that the non-observation of a time shifting interference pattern (or the fact that MSS observed a static interference pattern) was in fact positive evidence for the $\mathrm{AB}$ effect due to a subtle, almost cancellation between the magnetic $\mathrm{AB}$ phase shift and a phase shift coming from the $q\left(\frac{\mathbf{v}}{c} \times \mathbf{B}\right)$ force on 
the electrons. The Werner and Brill explanation of the non-observation of a time shifting interference pattern by MSS is conceptually similar to the explanation given in [2, 3] and by Eq. (21) and Eq. (3) above. In both cases, there is an interplay between the magnetic Aharonov-Bohm shifting of the phases and a dispersive shifting of the phases due to either an electric or magnetic field through which the electrons are moving. However, if one accepts both explanations then it seems that one will have over compensated for magnetic AB phase shift and that one should then again see a time shifting interference pattern (or the interference pattern would be washed out entirely). We now re-examine the Werner and Brill analysis and show that their cancellation is highly dependent on the strength of the unknown $60 \mathrm{~Hz}$ magnetic field in the MSS experiment, and in fact for certain magnetic field strengths one does not get the balancing of the $\mathrm{AB}$ phase shift against the shift due to the $q\left(\frac{\mathbf{v}}{c} \times \mathbf{B}\right)$ force.

To start the analysis we examine the re-produced figure 1 from [7]. In figure 1a, the electron beam is split into a diamond pattern by three crystals $C_{1}, C_{2}$ and $C_{3}$ in the absence of a magnetic field. In figure $1 \mathrm{a}$, the path length $l_{1}+l_{2}$ is equal to the path length of $m_{1}+m_{2}$ so - modulo the complication that the beams come together with a small angle $\varphi-$ one would get constructive interference. Next, in figure 1b, there is a uniform magnetic field of magnitude, $B_{0}$, coming out of the page. This bends the paths $l_{1}, l_{2}, m_{1}, m_{2}$ into circular arcs of radius

$$
R=\frac{2 \pi \hbar c}{e \lambda B_{0}} \approx \frac{850}{B_{0}} \text { gauss } \cdot \mathrm{cm} .
$$

In Eq. (10) we use gaussian units and in the last approximation we have plugged in the values of the constant $\hbar, c, e$ (in gaussian units) and we have taken the de Broglie wavelength of the electrons from [1] as $\lambda=4.86 \times 10^{-10} \mathrm{~cm}$.

Now, the analysis of [7] calculated the change in path length produced in the paths $l_{1}, l_{2}, m_{1}, m_{2}$ due to $B_{0}$ and then by dividing this path length difference by $\lambda / 2 \pi$ obtained a phase difference which when combined with the AB phase shift gave an almost cancellation. The path length differences $\Delta l_{2}=l_{2}^{\prime}-l_{2}$ and $\Delta m_{1}=m_{1}^{\prime}-m_{1}$ were both proportional to

$$
\Delta l_{2} \propto \frac{D^{2} \tan (\theta)}{R} \text { and } \Delta m_{1} \propto \frac{D^{2} \tan (\theta)}{R},
$$

where from Fig. 1 the angle $\theta$ is the crystal diffraction angle which for the set-up in [1] was $\theta \approx 2 \times 10^{-2}$ radians; $D$ was the distance between the crystals which for the set-up in [1] was varied between $5.0 \mathrm{~cm}$ and $3.49 \mathrm{~cm}$ (in subsequent estimates we take $D=5.0 \mathrm{~cm}$ ). The 


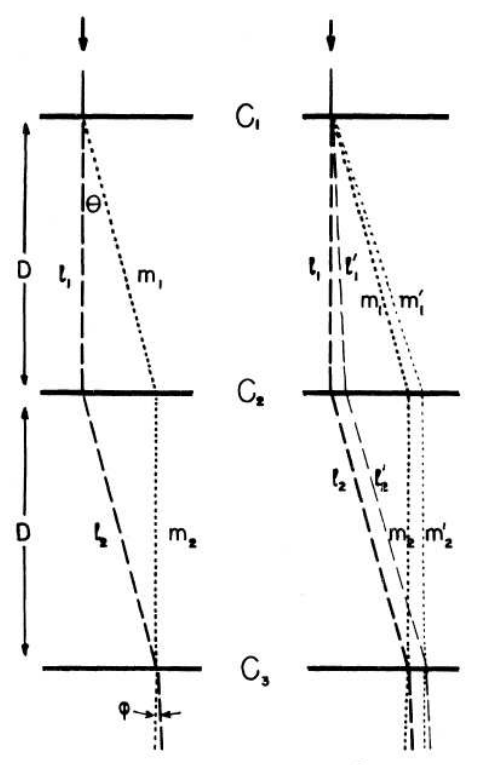

(a)

(b)

FIG. 1: The re-produced figure 1 of [7] showing the paths of the electrons in the interferometer. Figure $1 \mathrm{a}$ is without a magnetic field and figure $1 \mathrm{~b}$ has a uniform field $B_{0}$ coming out of the page. The three diffraction crystals, $C_{1}, C_{2}$ and $C_{3}$, are spaced a distance $D$ apart.

path length differences between difference $\Delta l_{1}=l_{1}^{\prime}-l_{1}$ and $\Delta m_{2}=m_{2}^{\prime}-m_{2}$ were ignored since as pointed out [7] these were of order $B_{0}^{2}$ whereas the path length differences $\Delta l_{2}$ and $\Delta m_{1}$ were of order $B_{0}$. In terms of $D$ and $R$ the path length differences $\Delta l_{1}$ and $\Delta m_{2}$ can be shown to have a proportionality

$$
\Delta l_{1} \propto \frac{D^{3}}{R^{2}}=\frac{D^{2}}{R} \frac{D}{R} \quad \text { and } \quad \Delta m_{2} \propto \frac{D^{3}}{R^{2}}=\frac{D^{2}}{R} \frac{D}{R} .
$$

The reason to split off one factor of $D / R$ in Eq. (12) is to make a comparison between these path length differences with the path length differences $\Delta m_{1}$ and $\Delta l_{2}$ from Eq. (11). The ratio $D / R$ is essentially the angle by which the electrons are bent by the magnetic field, $B_{0}$. In [7] this angle is called $\tau_{1}$ - more precisely the angle by which the path $m_{1}$ is bent is approximated as $\tau_{1} / 2$ [7]. Thus, both the path length differences $\Delta m_{1}$ and $\Delta l_{2}$ from Eq. (11) and the path length differences $\Delta m_{2}$ and $\Delta l_{1}$ are of the form $\frac{D^{2}}{R} \times$ angle, where angle is either the diffraction angle $\tan (\theta) \approx \theta$ from Eq. (11) or the deflection angle $\frac{D}{R}$ due to the magnetic field. The assumption made in [7], when ignoring the path length differences $\Delta l_{1}$ and $\Delta m_{2}$ relative to $\Delta l_{2}$ and $\Delta m_{1}$, is that $\frac{D}{R} \ll \theta$. Now, from [1] the diffraction angle was $\theta \approx 2 \times 10^{-2}$ radians and we take $D \approx 5.0 \mathrm{~cm}$. Now, even for a weak magnetic field of 
$B_{0}=1$ gauss (which is roughly only twice the Earth's magnetic field strength) Eq. (10) gives $R \approx 850 \mathrm{~cm}$ and thus $\frac{D}{R} \approx 6 \times 10^{-3}$ radians. Even for such a weak field the approximation $\frac{D}{R} \ll \theta$ does not really hold (it is true $\frac{D}{R}<\theta$ but the qualification "much less than" is not correct). For a magnetic field only 10 times stronger than the Earth's magnetic field, i.e., $B_{0}=5$ gauss, (10) gives $R \approx 170 \mathrm{~cm}$ and thus $\frac{D}{R} \approx 3 \times 10^{-2}$ radians for which the neither the relationship $\frac{D}{R} \ll \theta$ nor even $\frac{D}{R}<\theta$ is valid. Thus, depending on the strength of $B_{0}$ (which was small, but unspecified in the MSS experiment) the approximations used by Werner and Brill to ignore $\Delta l_{1}$ and $\Delta m_{2}$ might or might not be valid. For magnetic field greater than 1 gauss the approximation becomes quickly suspect. The result is that if one has a magnetic field even as weak as $B_{0} \approx 2$ gauss one should include $\Delta m_{2}$ and $\Delta l_{1}$ in calculating the total path length difference which is then $\left(\Delta l_{2}+\Delta l_{1}\right)-\left(\Delta m_{2}+\Delta m_{1}\right)$. Moreover, if the diffraction angle, $\theta$, is similar to the angle of deviation of the magnetic field, $\frac{D}{R}$, one finds that $\Delta l_{1} \sim \Delta m_{1} \sim \Delta l_{2} \sim \Delta m_{2}$ so that there is only a small total path length difference coming from the $q\left(\frac{\mathbf{v}}{c} \times \mathbf{B}\right)$ force. In this case the cancellation of the electric phase shift Eq. (2) with the usual magnetic phase shift Eq. (3) is crucial to explain the results of the MSS experiment. In any case ignoring the effect of the electric field for the time varying field is not valid while ignoring the effect of $q\left(\frac{\mathbf{v}}{c} \times \mathbf{B}\right)$ is justifiable depending on the strength of the unknown $B_{0}$.

In this paper, we have highlighted problems with previous explanations of and given an alternative explanation for the results of the MSS experiment [1], which was intended to study electron interference, and in fact an interference pattern was observed. However, later it was determined that the path of the electrons was contaminated with a $60 \mathrm{~Hz}$ "weak" magnetic field, but how weak was not specified. In light of the predictions for a quantum mechanical, AB phase shift [5, 6] which would have naively predicted a shifting/time-varying interference pattern in time to the temporal variation of the flux, the observations of a static interference pattern in [1] seemed, at first, to provide evidence against the $\mathrm{AB}$ phase shift. However, later experiments [4] with static magnetic fields from iron whiskers did confirm the AB phase shift. The results of the MSS experiment were explained in [7] as an almost cancellation between the $\mathrm{AB}$ phase shift and a phase shift coming from a path length difference produced by the $q\left(\frac{\mathbf{v}}{c} \times \mathbf{B}\right)$ force on the electrons since, unlike the ideal AB set-up, the electrons in the MSS experiment did move in a region with non-zero fields - both magnetic and electric. This was our first criticism of the previous analysis of the 
MSS experiment and all other studies of the time-dependent AB effect - the effect of the electric field, which arises from the time varying magnetic flux through Faraday's law, is completely ignored. Further, from our previous works [2, 3] and the calculations around Eqs. (2) and (3) above, we find that the phase shift coming from the electric field should exactly cancel the usual magnetic AB phase shift, without any need for the complicated geometrical constructions of [7]. Our second criticism is, depending on the strength of the "weak" magnetic field (which was not specified in either [1] or [7]) the approximation was made that the total path length difference was given by $\Delta l_{2}-\Delta m_{1}$ - thus ignoring the contributions of $\Delta l_{1}$ and $\Delta m_{2}$ to the total path length difference. This amounts to saying that the diffraction angle, $\theta$, was much larger than the angle of derivation due to the magnetic field, $\frac{D}{R}$, i.e., $\frac{D}{R} \ll \theta$. Using $D \approx 5.0 \mathrm{~cm}, \theta=2 \times 10^{-2}$ radians [1] and using Eq. (10) for $R$, we showed the even for $B_{0}$ of a few gauss that the approximation $\frac{D}{R} \ll \theta$ was not valid so that one should include $\Delta l_{1}$ and $\Delta m_{2}$ in the total path length difference. Including $\Delta l_{1}$ and $\Delta m_{2}$ would decrease the total path length difference since for $\frac{D}{R} \approx \theta$ one has $\Delta l_{1} \sim \Delta m_{1} \sim \Delta l_{2} \sim \Delta m_{2}$, which then gives only a small total path length difference. This would leave the proposed cancellation of the electric and magnetic phase shifts discussed in [2, 3] as the only explanation of the MSS experiment. Finally, the MSS experiment should be considered as an inadvertent test of the time-dependent $\mathrm{AB}$ effect rather than as a test of the time-independent $\mathrm{AB}$ effect. Here we are proposing that one should re-do a purposeful version of the time-dependent $\mathrm{AB}$ effect experiment to determine whether there is really a cancellation between the electric and magnetic phase as suggested in [2, 3], or if something like the resolution given in [7] is correct.

As a final comment we know of only one experiment which purposefully sought to test the time-dependent $\mathrm{AB}$ effect [10, 11], and this experiment did find no time varying shifting of the interference pattern in agreement with the prediction of [2, 3]. However the authors of [10, 11] later reported problems with the experimental set-up. This again calls for further experiments designed to specifically test the time-dependent AB effect to resolve this issue.

[1] L. Marton, J. A. Simpson, and J. A. Suddeth, Rev. Sci. Instr. 25, 1099 (1954).

[2] D. Singleton and E. Vagenas, Phys. Lett. B 723, 241 (2013). 
[3] J. MacDougall and D. Singleton, J. Math. Phys. 55, 042101 (2014).

[4] R.G. Chambers, Phys. Rev. Lett. 5, 3 (1960).

[5] Y. Aharonov and D. Bohm, Phys. Rev. 115, 484 (1959).

[6] W. Ehrenberg and R. E. Siday, Proc. Phys. Society B 62, 8 (1949).

[7] F.G. Werner and D. Brill, Phys. Rev. Lett. 4, 344 (1960).

[8] N.G. van Kampen, Phys. Lett. A 106, 5 (1984).

[9] K. Moulopoulos, J. Phys. A 43, 354019 (2010).

[10] Yu. V. Chentsov, Yu. M. Voronin, I. P. Demenchonok, and A. N. Ageev, Opt. Zh. 8, 55 (1996).

[11] A. N. Ageev, S. Yu. Davydov, and A. G. Chirkov, Technical Phys. Lett. 26, 392 (2000). 